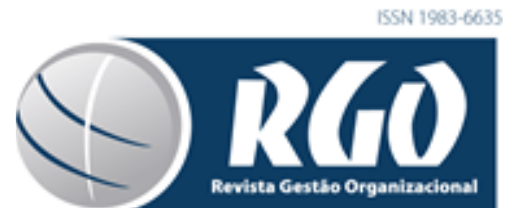

\title{
CARACTERÍSTICAS DOS CONSELHOS DE ADMINISTRAÇÃO E DESEMPENHO DAS FIRMAS BRASILEIRAS DE CAPITAL ABERTO
}

\section{BOARD OF DIRECTOR'S CHARACTERISTICS AND THE PERFORMANCE OF PUBLICLY TRADED BRAZILIAN FIRMS}

\author{
KELLEN LAZZARETTI \\ Doutoranda em Administração \\ Professora do SENAC/SC e da Universidade do Oeste de Santa Catarina \\ Orcid: http://orcid.org/0000-0003-3072-0888 \\ E-mail: kellen.lazzaretti29@gmail.com
}

Endereço: Avenida Nereu Ramos, 3777D, Bairro Seminário, CEP: 89813-000, Chapecó, Santa Catarina

\author{
ANDREZZA APARECIDA SARAIVA PIEKAS \\ Doutoranda em Administração \\ Universidade do Oeste de Santa Catarina \\ Orcid: http://orcid.org/0000-0002-9268-1506 \\ E-mail: andrezzapiekas@gmail.com \\ DULCIMAR JOSÉ JULKOVSKI \\ Doutorado em Administração \\ Universidade do Oeste de Santa Catarina \\ Orcid: https://orcid.org/0000-0003-2081-7213 \\ E-mail: professordulcimar@gmail.com
}

\begin{abstract}
RESUMO
Este estudo teve como objetivo investigar em que medida as características do conselho de administração influencia no desempenho das firmas brasileiras de capital aberto. A literatura é inconclusiva e indica que não existe uma relação única e definida. Nós argumentamos que características como tamanho, diversidade de gênero, independência do conselho e formação dos conselheiros podem influenciar o desempenho. Testamos nossas hipóteses em uma amostra de 364 firmas brasileiras de capital aberto listadas na B3, no período de 2015 a 2017. Os resultados sugerem que poucas são as características que, de fato influenciam no desempenho das empresas analisadas, tais como, o tamanho do conselho de administração e a presença feminina, influencia positivamente o desempenho. Entretanto, as demais características observadas não se mostraram significativas ao ponto de influenciar diretamente no desempenho. Nossos achados contribuem para a literatura sobre diversidade dos conselhos ao apontar evidências de que diversidade, não necessariamente implica em melhor ou pior desempenho, outras características de cunho conjuntural podem ser mais relevantes para indicar influência no desempenho. Esta constatação gera possibilidades de investigações futuras.
\end{abstract}

Palavras-chave: Conselho de administração. Diversidade. Desempenho da firma.

Data de submissão: 05/11/2019. Data de aceite: 04/12/2019. Data de publicação: 17/12/2019.

Artigo selecionado no fast track do COGECONT - International Conference in Management and Accounting, realizado na Universidade Comunitária da Região de Chapecó - Unochapecó, Chapecó - SC, de 17 a 19/10/2019. 


\begin{abstract}
This study aimed to investigate to what extent the characteristics of the board of directors influence the performance of publicly traded Brazilian firms. The literature is inconclusive and indicates that there is no single and definite relationship. We argue that characteristics such as size, gender diversity, board independence, and boardroom training can influence performance. We tested our hypotheses in a sample of 364 publicly traded Brazilian firms listed in B3, from 2015 to 2017. The results suggest that there are few features that actually influence the performance of the companies analyzed, such as the size of the board and the female presence positively influences the performance. However, the other characteristics observed were not significant to the point of directly influence performance. Our findings contribute to the literature on board diversity by pointing to evidence that diversity does not necessarily imply better or worse performance; other conjunctural characteristics may be more relevant to indicate influence on performance. This finding creates possibilities for future investigations.
\end{abstract}

Keywords: Board of director. Diversity. Firm's performance.

\title{
1 INTRODUÇÃO
}

Diante do turbulento cenário econômico brasileiro as firmas de capital aberto têm sofrido variações expressivas em seu desempenho. Tal condição faz com que os Conselhos de Administração (CA) ampliem seus esforços em prol da redução das incertezas de mercado e volatilidade do preço das ações, o que lhes garante maior legitimidade.

$O$ interesse pelas características do CA e sua influência no desempenho da firma têm sido amplamente discutido na literatura acadêmica (ADAMS; FERREIRA, 2009; GALBREATH, 2016; MAHADEO; SOOBAROYEN; HANUMAN, 2011; SILVA; MARTINS, 2015). Em geral, nestes estudos têm sido investigadas características como: tamanho (DARMADI, 2011; UJUNWA, 2012) e independência do CA (MAHADEO; SOOBAROYEN; HANUMAN, 2011), bem como, diversidade de gênero (ADAMS; FERREIRA, 2009; SILVA; MARTINS, 2015; MAHADEO; SOOBAROYEN; HANUMAN, 2011;), idade (MAHADEO; SOOBAROYEN; HANUMAN, 2011) e etnia (MARTINS; MAZER; LUSTOSA; PAULO, 2012) dos conselheiros, sob a perspectiva "racional" que a diversidade pode ser vista como uma característica "funcional" de um grupo de pessoas designadas para uma tarefa comum específica e tal funcionalidade pode levar a uma maior base de conhecimento, criatividade e inovação e, portanto, proporcionar vantagem competitiva à organização (MAHADEO; SOOBAROYEN; HANUMAN, 2011).

Neste artigo nos propomos a investigar em que medida as características do CA influenciam no desempenho das firmas brasileiras de capital aberto, uma vez que se trata de uma economia emergente. No Brasil estudos desta natureza são menos vastos, contudo, nos últimos anos a academia tem dado maior atenção para a problemática da diversidade dos CAs (LAZZARETTI; GODOI; CAMILO; MARCON, 2013; MARTINS; MAZER; LUSTOSA; PAULO, 2012; SILVA; MARTINS, 2015). Nosso estudo, no entanto, se diferencia dos demais por utilizar a amostra total de empresas listadas na B3.

Neste estudo sugerimos que características como tamanho, diversidade de gênero e independência do CA podem influenciar positivamente o desempenho da firma, enquanto a formação na área de gestão poderá ter uma influência negativa. 
Testamos nossas hipóteses usando uma amostra de 364 firmas brasileiras de capital aberto, no período de 2015 a 2017. Utilizamos dados disponibilizados na B3 e na Economatica. Os resultados evidenciam que poucas são as características que, de fato influenciam no desempenho das firmas analisadas. Especificamente, quanto maior o tamanho do CA, maior é o desempenho das firmas bem como a presença de mulheres influencia positivamente no desempenho das firmas.

Nosso estudo contribui para a literatura sobre diversidade dos CAs ao indicar que diversidade, não necessariamente implica em melhor ou pior desempenho, outras características de cunho conjuntural podem ser mais relevantes para indicar desempenho. Esta constatação gera possibilidades de investigações futuras.

\section{REVISÃO DA LITERATURA E DESENVOLVIMENTO DAS HIPÓTESES}

As firmas têm na sua essência a busca contínua por melhor desempenho. O CA, considerado um dos principais agentes da governança corporativa, tem entre suas responsabilidades, o papel de fiscalizador da atuação do agente em nome dos acionistas, assim fomentando o bom desempenho e a maximização do valor da firma (BANGE; MAZZEO, 2004).

No Brasil, as características gerais do CA, que devem ser observadas pelas companhias de capital aberto, estão dispostas na Lei 6.404/76. Adicional à legislação, o Instituto Brasileiro de Governança Corporativa (IBGC) dissemina o Código das Melhores Práticas de Governança Corporativa (IBGC, 2009), o qual faz recomendações acerca de características desejáveis a serem adotadas por tais firmas, com o intuito de atribuir maior transparência e responsabilidade aos acionistas e interessados em geral. Observa-se que os CAs seguem normas claras, o que os torna cada vez mais similares (DIMAGGIO; POWELL, 1983).

No âmbito desta pesquisa, estamos interessados em investigar em que medida, as características do CA influenciam o desempenho da firma. Para isso, quatro variáveis independentes são analisadas: tamanho do conselho (número de conselheiros), gênero, formação do presidente e vice-presidente e independência do conselho (número de conselheiros independentes) como uma representação do conceito geral de sua composição. Como variáveis moderadoras foram utilizadas proporção de mulheres no conselho e gênero do presidente e vice-presidente. Consequentemente, esta seção revisa estudos que examinaram a relevância dessas variáveis sob o desempenho da firma. Como variáveis de controle foram utilizados o setor de atuação e a diversificação de atividades das firmas analisadas.

\subsection{DESEMPENHO DA FIRMA}

Resumidamente, o desempenho financeiro indica a capacidade de geração de resultado financeiro, mais especificamente criação de valor face aos investimentos realizados (MARTINS et al. 2012; DEGENHART; VOGT; KAVESKI; FANK; SCARPIN, 2017). O desempenho aponta como anda a saúde financeira de uma firma ao longo de determinado período de tempo. É comum que o desempenho sirva de base para comparações entre setores, seja da própria firma ou de firmas similares (GOES; MARTINS; MACHADO, 2017).

Habitualmente, o conceito de desempenho financeiro está relacionado com informação contábeis, oriundas das demonstrações financeiras. Entretanto, a literatura aponta várias formas de mensurar desempenho financeiro da firma, três são mais usuais. $A$ primeira é relacionada a indicadores de mercado, a segunda atrelada à relatórios contábeis, e 
a terceira combina aspectos mercadológicos e contábeis (MERCHANT, 2006). No ambiente corporativo os relatórios contábeis apresentam-se como uma ferramenta fundamental para a avaliação de desempenho, a amplitude de informações e a transparência deste tipo de relatório, permite aos interessados verificar a rentabilidade da firma (BEKANA; ABITIE, 2012).

Vários são os estudos que relacionam o desempenho da firma e as características do CA, a partir de relatórios contábeis. Muitos deles apontam para uma relação positiva (ADAMS; FERREIRA, 2009; AKPAN; AMRAN, 2014; WAHBA, 2015; GALBREATH, 2016; JEDI; NAYAN, 2018). Enquanto outros apresentam a relação inversa, como por exemplo os estudos de Linck, Netter e Yang (2008); Guest (2008); Singh e Gaur (2009) e Khosa (2017).

Neste estudo, adotamos a mensuração de desempenho a partir de dados extraídos de relatórios contábeis, que segundo Merchant (2006) são os mais utilizados por possuírem vantagem frente aos demais. Mensuramos o desempenho a partir da média do retorno sobre o ativo (ROA) das firmas listadas na B3 nos anos de 2015, 2016 e 2017. Assim como os estudos de Ahmadi, Naka e Bouri (2018), Bennouri, Chtioui, Nagati e Nekhili (2018) e Hakimi, Rachdi, Ben Selma Mokni e Hssini (2018) optamos por utilizar o ROA por ser uma medida de desempenho que sofre menor influência de variáveis macroeconômicas.

\subsection{TAMANHO DO CA}

Os CAs são o principal mecanismo de ligação entre as firmas e seus ambientes externos (PFEFFER; SALANCIK, 1978), por este motivo, ampliam-se as investigações sobre o tamanho do conselho e se este afeta ou não o desempenho da firma. Um dos primeiros estudos empíricos realizados para verificar os efeitos do tamanho do CA sobre o desempenho da firma foi realizado por Yermack (1996). Posteriormente muitos outros estudos foram realizados, sendo que não há consenso sobre a direção dessa relação.

Muitos estudos encontraram uma relação positiva entre o tamanho do $C A$ e o desempenho da firma (ADAMS; FERREIRA, 2009; DALTON; DAILY; ELLSTRAND; JOHNSON, 1998; DARMADI, 2011; PEARCE; ZAHRA, 1992; UJUNWA, 2012). Os achados de Akpan e Amran (2014) e Gaur, Bathula e Singh (2015) indicam que maior número de membros no CA é beneficiário para a firma, em função de várias situações, tais como a captação de recursos, o alargamento de competências, experiências e conhecimentos, que por sua vez, contribuem para um melhor desempenho. Corrobora Wahba (2015) ao afirmar que é plausível supor que, quanto maior for o número de membros do conselho, melhor será o desempenho.

Alternativamente, estudos documentam a existência de uma relação inversa entre o tamanho do conselho e o desempenho da firma (GUEST, 2008; JENSEN, 1993; REDDY; LOCKE; SCRIMGEOUR; GUNASEKARAGE, 2008; VAN DEN BERGHE; LEVRAU, 2004; YERMACK, 1996). Para Jensen (1993) à medida que os conselhos crescem, tornam-se menos propensos a funcionar de forma eficaz, pois crescem também os problemas de coordenação e governança. O estudo de Jensen (1993) é respaldado pelos achados de Yermack (1996) que concluiu que pequenos CAs são mais eficazes, em função das facilidades de comunicação e agilidade na tomada de decisões.

Embora tenham sido encontrados resultados distintos, argumentamos que, o maior número de membros no CA implica em acesso a uma gama mais ampla de recursos, que por sua vez, contribuem para um melhor desempenho da firma. Logo, a primeira hipótese que propomos é:

Hipótese 1 - O tamanho do CA influencia positivamente no desempenho da firma. 


\subsection{DIVERSIDADE DE GÊNERO}

O cenário de desigualdade de gênero no Brasil está presente nos CAs. Segundo os dados da pesquisa Perfil dos Conselhos de Administração, lançada pelo Instituto Brasileiro de Governança Corporativa, no segundo semestre de 2016, as mulheres representavam apenas $7,9 \%$ dos conselheiros nas firmas listadas em bolsa (IBGC, 2016). Comparando este dado com demais países observa-se que o Brasil está com um número inferior à média de $12 \%$ encontrada em 49 países analisados pela Catalyst (2018). A Europa é o continente com maior percentual, $25 \%$ em média, que tem a Noruega em primeiro $(40,1 \%)$, seguido pela Suécia $(33,7 \%)$ e França $(33,5 \%)$ (CATALYST, 2018). Os países com maiores índices de mulheres nos conselhos, como Noruega e Suécia, adotaram cotas e metas para aumentar os índices de mulheres nos conselhos. A Suécia, por exemplo, teve quase o dobro da porcentagem média de mulheres em conselhos (cerca de $34 \%$ ) do que países sem essas medidas (cerca de $18 \%$ ) (CATALYST, 2018).

No Brasil, a Comissão de Defesa dos Direitos da Mulher aprovou em 2017 o Projeto de Lei 112/2010 que exige a presença de $40 \%$ de mulheres nos CAs das firmas públicas, sociedades de economia mista e demais firmas em que a União, direta ou indiretamente, detenha a maioria do capital social com direito a voto. Pela proposta, o percentual passaria a valer a partir de 2022 e seria preenchido gradualmente (BRASIL, 2019).

$\mathrm{Na}$ literatura a questão de gênero é uma das características do CA mais debatidas, a exemplo de Burke $(1997,2003)$ que relata os benefícios da presença de mulheres nos CA, contudo, outros estudos também indicam que um maior número de mulheres no conselho tem um efeito positivo no valor das firmas (CAMPBELL; MÍNGUEZ-VERA, 2008) melhora o comprometimento ao comparecimento e alocam mais esforços ao monitoramento nas firmas (ADAMS; FERREIRA, 2009) a representação das mulheres nos CAs está positivamente ligada à responsabilidade social corporativa (GALBREATH, 2016) aumenta significativamente o ROA e o ROE (BENNOURI et al, 2018; JEDI; NAYAN, 2018).

A Catalyst (2004) identificou que as firmas que tinham mais mulheres nos conselhos tiveram melhores resultados financeiros como $16 \%$ mais retorno sobre vendas e $26 \%$ de retorno sobre o capital investido. Galbreath (2016) sugere que existe a possibilidade de que as mulheres nos CAs possam afetar positivamente o desempenho financeiro, mas que seu impacto positivo seja mediado.

Simpson, Carter e D'Souza (2010) observam que há razões lógicas para esperar uma relação positiva entre mulheres no CA e um lucro maior e melhor retorno para os acionistas, no entanto, existem estudos que comprovam não haver ou até mesmo haver uma relação negativa entre mulheres no conselho e desempenho financeiro. Silva e Martins (2015) conduziram uma pesquisa utilizando uma amostra composta pelas firmas brasileiras mais líquidas listadas na Bolsa de valores do Brasil, nos períodos de 2010 a 2013 e identificaram que a relação entre gênero feminino e desempenho financeiro destas firmas foi positiva, porém, sem significância estatística. Diante destes dados, nós consideramos que,

Hipótese 2a - A presença feminina no CA influencia positivamente o desempenho da firma.

Estudo realizado no Brasil com 28 bancos listados na Bolsa de valores do Brasil sugere que a presença das mulheres entre os membros do CA dessas firmas tem uma relação direta, embora negativa, com o desempenho financeiro, em que cada membro do gênero feminino a mais que integra o CA tende a reduzir seu ROI em 2,2\% (MARTINS; MAZER; LUSTOSA; PAULO, 2012). Contudo, Lee, Marshall, Rallis e Moscardi (2015) identificaram que três ou mais 
mulheres mudam substancialmente a dinâmica do conselho e potencializam a opinião das mulheres. Também que firmas com "forte liderança feminina" (principalmente mulheres em conselhos) estavam correlacionadas com maior Retorno sobre o Patrimônio Líquido (ROE) do que as firmas sem, bem como um preço mais alto de suas ações. Lee et al. (2015) também identificaram que as firmas com menos mulheres nos conselhos tiveram mais controvérsias relacionadas à governança do que a média.

Para colher os benefícios da diversidade, os CAs precisam de pelo menos três ou mais mulheres para criar uma "massa crítica", o que pode levar a um melhor desempenho financeiro (KONRAD; KRAMER; ERKUT, 2008). Joecks, Pull e Vetter (2013) encontraram evidências em firmas alemãs que a diversidade de gênero afeta negativamente o desempenho da firma e, somente com a presença de minimamente três mulheres, estaria associada a um desempenho empresarial mais alto do que os conselhos masculinos. Assim sendo sugerimos que,

Hipótese $2 \mathrm{~b}-\mathrm{A}$ proporção de mulheres no $\mathrm{CA}$ modera positivamente a relação entre $a$ presença feminina no conselho e o desempenho da firma.

Estudos conduzidos pelo IBGC em 2009, 2010 e 2011 mostraram que 4,92\%, 3,27\% e $3,9 \%$ respectivamente dos presidentes dos conselhos das firmas brasileiras listadas na Bolsa de valores do Brasil eram mulheres. Lazzaretti (2011), por sua vez, identificou 24 firmas brasileiras listadas na Bolsa de valores do Brasil com mulheres ocupando assento de presidente ou vice-presidente dos conselhos, o que equivale à $5,4 \%$, contudo, se consideradas apenas as presidentes, havia nove mulheres $(2,2 \%)$, sendo que dessas, seis são membros da família controladora (Lazzaretti, 2011).

Pesquisa conduzida pela Catalyst (2011) mostrou o panorama de participação feminina na presidência de conselhos de diversos países que, tinha Noruega (12\%), Turquia $(11,1 \%)$, Polônia $(6,7 \%)$ com os maiores percentuais e Reino Unido e França (2\%), Suiça (1,9\%) e Alemanha (1,2\%) com os menores índices. Observa-se que o Brasil está entre os países com menor índice (2,2\%). Muito embora a presença das mulheres na presidência ou vicepresidência do CA seja baixa, consideramos que,

Hipótese 2c - A presença de mulher na presidência ou vice-presidência do CA modera positivamente a relação entre a presença feminina no conselho e o desempenho da firma.

\subsection{FORMAÇÃO DO PRESIDENTE E VICE-PRESIDENTES DO CONSELHO}

Quanto ao perfil profissional dos conselheiros Mahadeo, Soobaroyen e Hanuman (2011) entendem que um conselho mais diversificado em termos de formação é mais favorável para a firma uma vez que evita a formação de clãs, contudo, estudos anteriores realizados no Brasil não evidenciam esta diversificação de formação dos conselheiros. Bruère, Silva e Santos (2007) identificaram que firmas nos setores de Alimentos \& Bebidas e Energia Elétrica da Bolsa de valores do Brasil as formações mais frequentes dos conselheiros eram: Engenharias (35,9\%), Direito (17,7\%), Administração $(16,8 \%)$, Economia $(16,1 \%)$, Contabilidade $(5,2 \%)$ e as demais profissões em conjunto somavam $8,3 \%$. Já Martins et al (2012) encontrou que as formações mais comuns entre os conselheiros dos bancos brasileiros, também listados na Bolsa de valores do Brasil eram: Economia (25\%), Engenharias diversas $(14,4 \%)$, Administração (13,3\%), Direito (8,0\%), Contabilidade $(4,3 \%)$ e Outros $(35,0 \%)$. 
Os dados de formação dos conselheiros brasileiros mostram uma homogeneidade, uma vez que a formação da maioria é na área de gestão (economia, contabilidade e administração), engenharias ou direito.

Neste sentido, entendemos que,

H3 - Presidentes ou vice-presidentes do CA com formação na área de gestão influenciam negativamente o desempenho da firma.

\subsection{INDEPENDÊNCIA DO CONSELHO}

Usualmente, na maioria dos países os códigos de governança corporativa exigem que os CAs de firmas de capital aberto tenham uma combinação de membros internos e externos (JACKLING; JOHL, 2009). No Brasil o Regulamento de Práticas Diferenciadas de Governança Corporativa da B3 exige que as firmas dos segmentos Novo Mercado e Nível 2 tenham participação de membros independentes nos CAs (MARTINS, et al. 2012). Teoricamente, há duas linhas de investigação, uma aponta que há uma relação positiva entre a independência do conselho e o desempenho da firma e outra, documenta uma relação negativa entre a independência do conselho e o desempenho da firma.

Há evidências de que a maior independência do conselho leva a um melhor monitoramento das decisões gerenciais (FAMA; JENSEN, 1983; WEISBACH, 1988) reduz os conflitos entre as partes interessadas (ANDERSON; REEB, 2003; CHEN; ROBERTS, 2010; DUCHIN; MATSUSAKA; OZBAS, 2011) e podem, portanto, gerar um efeito positivo no desempenho da firma. Estudos apontam que um conselho mais independente diminui o custo do financiamento da dívida (PATHAN; FAFF, 2013), aumenta a classificação de crédito da firma e reduz o risco associado ao custo de capital (ASHBAUGH-SKAIFE; COLLINS; LA FOND, 2006). Diretores independentes têm maior autoridade de supervisão (CHAU; GRAY, 2010), desta forma, favorecem que o desempenho de firmas com maior proporção de diretores independentes seja melhor do que firmas com menor proporção de diretores independentes (ALI; NAKAA; ABDELFETTAH, 2018; ERTIMURA; FERRIB; STUBBENC, 2010).

Em contrapartida, a literatura apresenta estudos que encontraram uma relação negativa entre o desempenho da firma e a proporção de conselheiros independentes (KHOSA, 2017; SINGH; GAUR, 2009). Kiel e Nicholson (2003) destacam que os proponentes da teoria da mordomia sustentam que o desempenho superior estará ligado à maioria dos conselheiros internos. Linck, Netter e Yang (2008) relatam que os conselheiros independentes são incompetentes para controlar efetivamente os líderes, e isso implica necessariamente em desempenho inferior. Embora alguns estudos tragam argumentos contrários, nós argumentamos que,

H4 - A proporção de conselheiros independentes influencia positivamente no desempenho da firma.

Na Figura 1 é representado o modelo proposto. 
Figura 1- Modelo proposto

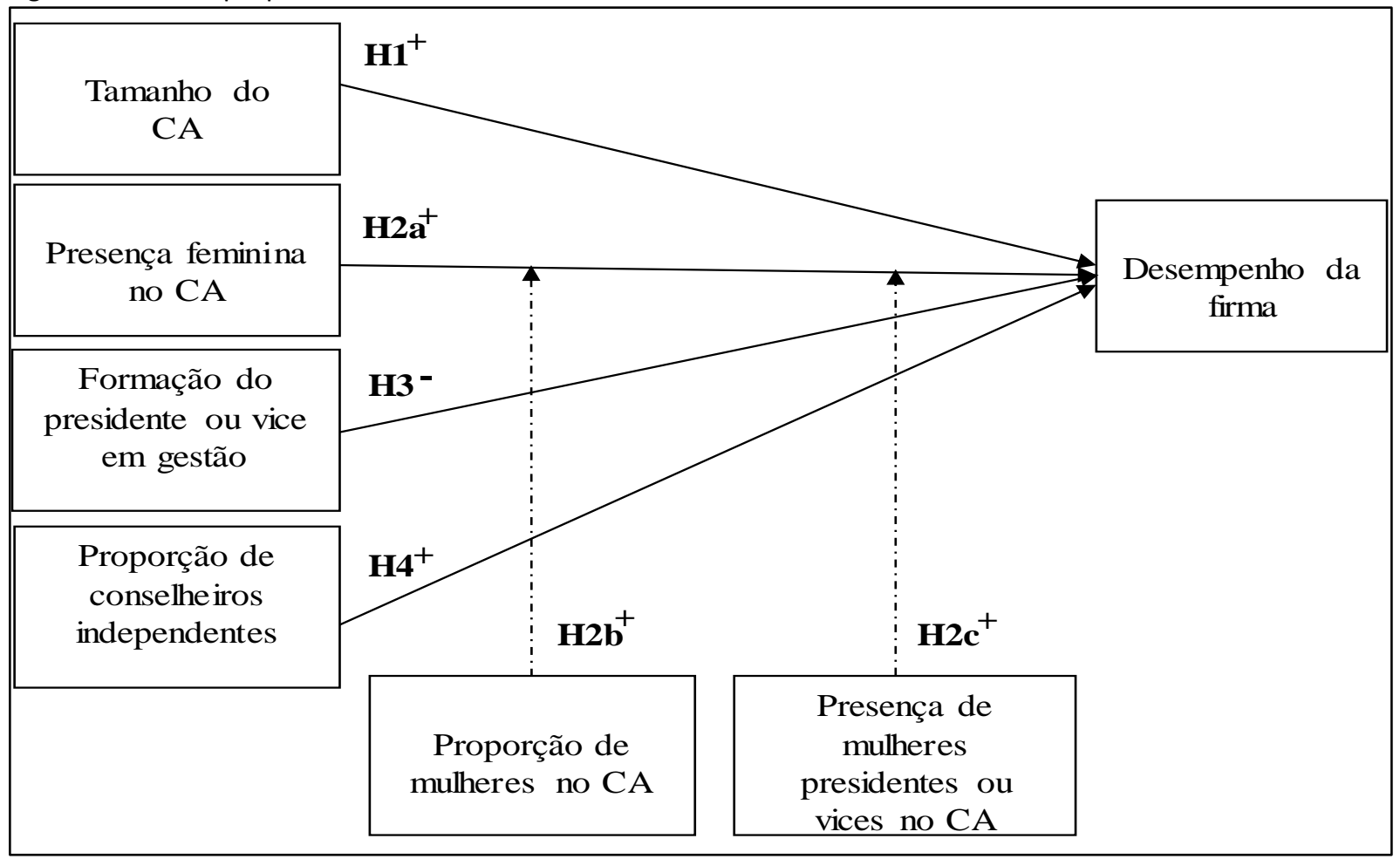

Fonte: Elaborado pelos autores (2019).

\section{PROCEDIMENTOS METODOLÓGICOS}

Este estudo enfoca o caso do Brasil, mais especificamente nas firmas de capital aberto listadas na bolsa de valores brasileira (B3). A B3 é a bolsa de valores oficial do Brasil, sediada na cidade de São Paulo, em 2017 era a quinta maior bolsa de mercado de capitais e financeiro do mundo, com patrimônio de 13 bilhões de dólares (CVM, 2018).

\subsection{DADOS E AMOSTRA}

Para definir a amostra adotamos os seguintes procedimentos. Primeiro selecionamos todas as firmas listadas no próprio site da B3, que somaram 435 empresas, posteriormente excluímos aquelas, cujo formulário de referência não estava disponível. Destes formulários de referência foram extraídos dados referentes ao setor de atuação da firma e características do CA restando 418 firmas. Por fim, excluímos as firmas que não tinham disponíveis os dados referentes ao retorno sobre o ativo (ROA) dos anos 2015, 2016 e 2017. Os dados referentes ao ROA foram coletados na base Economática, restando 364 firmas. Destas 364 firmas foram ainda excluídas 33, considerados outliers. A amostra final compreendeu 331 firmas listadas na B3, em 2018.

O formulário de referência como fonte de dados têm sido amplamente utilizados para estudos, como por exemplo, Três, Serra e Ferreira (2004) que analisaram a influência do CEO no desempenho de organizações listadas na B3, Degenhart et al. (2017) analisaram fatores que determinam o desempenho de firmas familiares brasileiras de capital aberto (GOES; MARTINS; MACHADO, 2017) que buscaram compreender as diferenças entre o desempenho de firmas familiares listadas em bolsa frente a firmas não familiares. 


\subsection{VARIÁVEIS}

A variável dependente é desempenho medido a partir de dados extraídos de relatórios contábeis, que segundo Merchant (2006) são os mais utilizados por possuírem vantagem frente aos demais. Mensuramos o desempenho considerando a média do retorno sobre o ativo (ROA) das firmas listadas na B3, nos anos de 2015, 2016 e 2017. Assim como os estudos de Ahmadi, Naka e Bouri (2018), Bennouri, Chtioui, Nagati e Nekhili (2018) e Hakimi, Rachdi, Ben Selma Mokni e Hssini (2018) optamos por utilizar o ROA por ser uma medida de desempenho que sofre menor influência de variáveis macroeconômicas.

\subsection{VARIÁVEIS INDEPENDENTES}

Tamanho do conselho foi medido a partir da contagem do número de conselheiros efetivos, incluindo seu presidente e vice-presidente. Mensuramos a diversidade de gênero observando se a firma tinha membros do gênero feminino em seu conselho, constituindo uma variável binária caracterizada dicotomicamente 1 para homem e 0 para mulher. Na sequência foi calculada a proporção de mulheres a partir da divisão do número de mulheres e do número total de membros efetivos de cada conselho, por fim, outra variável binária, que indicava a existência de mulher ocupando o cargo de presidente ou vice-presidente do conselho foi estabelecida sendo que para mulher na presidência ou vice-presidência foi atribuído 1 para sim e 0 para não.

Para análise da formação do presidente e vice-presidente do conselho consideramos as informações auto declaradas no resumo do currículo do conselheiro. Inicialmente descrevemos a formação por estes indicadas, na sequência formamos três categorias, sendo classificadas como 1 aqueles com formação na área de Gestão, que envolveu a formação em Administração, Ciências Contábeis, Ciências Econômicas; 0 aqueles com formação em outras áreas do conhecimento, sendo as mais comuns as Engenharias e Direito. Para aqueles sem formação informada foi atribuído o número 2. Para teste da hipótese 3 consideramos a formação ou não na área de gestão. A independência do conselho foi medida a partir da contagem do número de conselheiros independentes e posteriormente calculada a proporção sobre o número de conselheiros efetivos incluindo presidente e vice-presidente de cada firma.

\subsection{VARIÁVEIS DE CONTROLE}

Como variáveis de controle utilizamos o setor principal de atuação da firma. Esta informação foi oriunda de duas fontes distintas, a primeira a declarada no cadastro da firma junto a B3 e confrontado com aquela informada na Classificação Nacional de Atividades Econômicas (CNAE) da Receita Federal do Brasil. Desta análise emergiram 20 categorias setoriais distintas. Estas informações permitiram também controlar a diversificação de atuação da firma. Um controle para saber se a firma exerce atividades diversificadas foi incluso, sendo codificada 1 para firmas com atuação diversificada e 0 para firmas que atuam em apenas um setor.

\subsection{ANÁLISE DE DADOS}

Para análise dos dados, foram utilizadas técnicas estatísticas de correlação e regressão linear, com utilização do software SPSS (Statistical Package for the Social Science), versão 24. Estas ferramentas de análise de dados quantitativos têm como objetivo medir, explicar e prever o grau de relação das variáveis e, não somente no número de observações (HAIR; BLACK; BABIN; ANDERSON; TATHAN, 2009). 


\section{ANÁLISE DOS RESULTADOS}

A análise de associação dos fatores e as estatísticas descritivas podem ser observadas na Tabela 1. As correlações não são elevadas a ponto de implicar em multicolinearidade, todos os fatores de variância (VIF) ficaram abaixo de 3. A sumarização dos dados da regressão pode ser evidenciada na Tabela 2. As hipóteses são testadas em oito modelos. No modelo 1 são testadas apenas variáveis de controle, que por sua vez explicam apenas $0,2 \%$ do desempenho. Os modelos 2 a 7 testam as hipóteses propostas individualmente, enquanto o modelo 8 é o modelo completo, que serve de base para nossa análise.

A hipótese 1 pressupõe que o tamanho do conselho tem um impacto positivo no desempenho da firma. A amostra de empresas analisadas apresentou um tamanho médio de seis conselheiros, sendo que o menor conselho é formado por apenas um conselheiro, enquanto o maior, é formado com 15 conselheiros. No teste de regressão encontramos significância a um nível de $0,004(p<0,01)$ indicando que tamanho do conselho tem um impacto positivo no desempenho da firma, o que dá suporte para $\mathrm{H} 1$.

A hipótese 2 a propõem que a presença feminina no CA tem um impacto positivo no desempenho, o que é confirmado pelo nível de significância encontrado de 0,015 ( $p<0,05)$, suportando assim H2a. Também encontramos significância para a hipótese $2 \mathrm{~b}$ que supunha que a proporção de mulheres no CA modera positivamente o impacto entre a presença de mulheres no conselho e desempenho, esta hipótese foi suportada apresentando nível de significância de $0,068(p<0,1)$. Entretanto cabe ressaltar que no caso das empresas analisadas, o percentual de mulheres não ultrapassa 7\%, número este considerado baixo se comparado às estatísticas internacionais. Já a hipótese 2 c propunha que o gênero do presidente ou vicepresidente do conselho, se feminino, modera positivamente a relação entre a presença feminina no conselho e o desempenho da firma, contudo esta hipótese foi refutada.

A hipótese 3 afirma que presidentes ou vice-presidentes do CA com formação na área de gestão tem impacto negativo no desempenho da firma, uma vez que uma maior diversidade de formação dos membros do conselho, pode ser benéfico para a tomada de decisão, o que pode impactar em um melhor desempenho. Nossos resultados, no entanto, refutam a hipótese, pois o teste de hipótese não apresentou significância estatística. A hipótese 4 pressupunha que a proporção de conselheiros independentes tem um impacto positivo no desempenho da firma, contudo, esta hipótese foi rejeitada.

O Quadro 1 resume as informações dos testes de hipótese e seus resultados.

Quadro 1- Resultado dos testes de Hipótese

\begin{tabular}{|l|c|}
\hline \multicolumn{1}{|c|}{ Hipótese } & Posição \\
\hline H1 - O tamanho do CA influencia positivamente no desempenho da firma. & Aceita \\
\hline H2a - A presença feminina no CA influencia positivamente o desempenho da firma. & Aceita \\
\hline $\begin{array}{l}\text { H2b - A proporção de mulheres no CA modera positivamente a relação entre a presença } \\
\text { feminina no conselho e o desempenho da firma. }\end{array}$ & Aceita \\
\hline $\begin{array}{l}\text { H2c - A presença de mulher na presidência ou vice-presidência do CA modera } \\
\text { positivamente a relação entre a presença feminina no conselho e o desempenho da firma. }\end{array}$ & Rejeitada \\
\hline $\begin{array}{l}\text { H3 - Presidentes ou vice-presidentes do CA com formação na área de gestão influenciam } \\
\text { negativamente o desempenho da firma. }\end{array}$ & Rejeitada \\
\hline $\begin{array}{l}\text { H4 - A proporção de conselheiros independentes influencia positivamente no desempenho } \\
\text { da firma. }\end{array}$ & Rejeitada \\
\hline
\end{tabular}

Fonte: Elaborado pelos autores (2019). 
EDIÇÃO ESPECIAL: COMEMORATIVA AOS 45 ANOS DOS CURSOS DE GRADUAÇÃO EM CIÊNCIAS CONTÁBEIS E ADMINISTRAÇÃO DA UNOCHAPECÓ

Tabela 1- Estatísticas descritivas e correlação

\section{CORRELAÇÃO}

\begin{tabular}{|c|c|c|c|c|c|c|c|c|c|c|c|c|}
\hline & & & & & & & & & & & \multicolumn{2}{|c|}{ 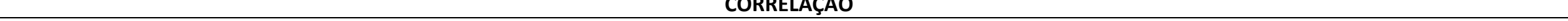 } \\
\hline 1 & Média ROA & 4.12 & 8,49 & 1 & & & & & & & & \\
\hline 2 & Tamanho do CA & 6,16 & 2,50 &,- 102 & 1 & & & & & & & \\
\hline 3 & Mulheres no CA & 0,43 & 0,50 & 0,071 & $173^{* *}$ & 1 & & & & & & \\
\hline 4 & Formação do presidente e ou vice na área de gestão & 0,53 & 0,50 & 0,006 & $142^{*}$ &, 046 & 1 & & & & & \\
\hline 5 & Proporção de conselheiros independentes no CA & 22,06 & 24,52 &,- 023 & $366^{* *}$ &,- 016 & $162^{* *}$ & 1 & & & & \\
\hline 6 & Mulheres no CA x Proporção de Mulheres no CA & 11,06 & 16,84 &, 044 &,$- 171^{* *}$ &, $758^{* *}$ & ,043 &,$- 132^{*}$ & 1 & & & \\
\hline 7 & Mulheres no CA x Gênero do presidente e vice-presidente & 0,30 & 0,46 & 034 &, $312^{* *}$ & $791^{* *}$ & ,066 & ,067 & $454^{* *}$ & 1 & & \\
\hline 8 & A firma atua em setores diversos & 0,59 & 0,49 &,- 040 &,$- 116^{*}$ &,- 105 &,$- 133^{*}$ &,- 013 &,- 090 &,- 006 & 1 & \\
\hline 9 & Setor principal & 9,87 & 4,25 & ,019 &,- 040 &,- 003 &, $152^{*}$ & ,028 & ,011 & ,035 & 105,- & 1 \\
\hline
\end{tabular}

Fonte: Dados da pesquisa (2019).

Tabela 2 - Regressão

\begin{tabular}{|c|c|c|c|c|c|c|c|c|}
\hline \multicolumn{9}{|c|}{ REGRESSÃO } \\
\hline & Mod. 1 & Mod. 2 & Mod. 3 & Mod. 4 & Mod. 5 & Mod. 6 & Mod. 7 & Mod. 8 \\
\hline \multicolumn{9}{|l|}{ Variável dependente } \\
\hline \multicolumn{9}{|l|}{ Desempenho (ROA) } \\
\hline \multicolumn{9}{|l|}{ Variáveis independentes } \\
\hline Tamanho do CA & & 0,420 & & & & & & $0,004 * *$ \\
\hline Há mulheres no CA & & & 0,237 & & & & & $0,015^{*}$ \\
\hline Formação do presidente e ou vice na área de gestão & & & & 0,937 & & & & 0,584 \\
\hline Proporção de conselheiros independentes no CA & & & & & 0,612 & & & 0,787 \\
\hline Há mulheres no CA x Proporção de Mulheres no CA & & & & & & 0,493 & & $0,068+$ \\
\hline Há mulheres no CA x Gênero do presidente e vice-presidente & & & & & & & 0,601 & ,358 \\
\hline \multicolumn{9}{|l|}{ Variáveis de controle } \\
\hline A firma atua em setores diversos & 0,420 & 0,283 & 0,503 & 0,704 & 0,414 & 0,461 & 0,519 & 0,663 \\
\hline Setor principal & 0,801 & 0,887 & 0,787 & 0,686 & 0,791 & 0,801 & 0,707 & 0,791 \\
\hline 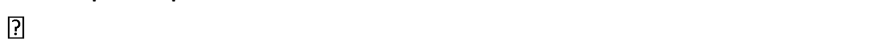 & 4,357 & 7,014 & 3,772 & 4,165 & 4,566 & 4,105 & 4,047 & 7,813 \\
\hline R2 & 0,002 & 0,015 & 0,007 & 0,001 & 0,003 & 0,004 & 0,003 & 0,049 \\
\hline $\mathrm{N}$ & 331 & 331 & 331 & 266 & 331 & 331 & 316 & 266 \\
\hline
\end{tabular}

Fonte: Dados da pesquisa (2019). 


\section{CONSIDERAÇÕES FINAIS}

Os estudos que avaliam características dos conselhos de administração e sua influência no desempenho da firma são vastos, principalmente em países europeus, onde o debate sobre diversidade é mais avançado. No Brasil, contudo, apesar do IBGC indicar as boas práticas de governança corporativa, observa-se que, em certa medida, a diversidade nos conselhos de administração é um esforço para demonstrar uma ausência de discriminação (ERHARDT; WERBEL; SHRADER, 2003).

A literatura não é clara se essa diversidade influencia o desempenho organizacional. Os trabalhos de Bennouri et al. (2018) e Jedi e Nayan (2018) mostraram que há uma relação positiva entre diversidade do conselho e desempenho, enquanto Martins et al. (2012) e Joecks, Pull e Vetter (2013) identificaram que não há relação ou até que há uma relação negativa. Estes argumentos teóricos enfatizam a carência de uma melhor investigação teórica e empírica, principalmente no caso brasileiro, pouco explorado. Neste sentido este estudo foi construído a fim de eliminar esta lacuna, ao passo que investigou em que medida as características do CA influenciam o desempenho das firmas brasileiras listadas na B3.

Nosso estudo amplia tal entendimento e sugere que no caso das firmas brasileiras analisadas, poucas são as características que influenciam o desempenho da firma. Descobrimos que o tamanho do CA e a presença feminina influencia positivamente 0 desempenho. Descobrimos também que a proporção de mulheres modera positivamente a relação entre a presença feminina e o desempenho, o que vem ao encontro dos estudos de Konrad, Kramer e Erkut (2008), Joecks, Pull e Vetter (2013) e Lee, Marshall, Rallis, Moscardi, (2015).

Entretanto as demais características observadas não se mostraram significativas ao ponto de influenciar diretamente no desempenho. Isso pode ser em partes explicado pelo alto isomorfismo presente nestas firmas que concentram um número reduzido de mulheres e de conselheiros independentes, privilegiando uma homogeneidade de características que se resumem em conselheiros do gênero masculino, diretamente ligados às firmas e com formação nas áreas de gestão, engenharia e direito, independente do setor de atuação ou da segmentação de mercado.

Este estudo nos permite afirmar que a mera inclusão de diversidade nos conselhos não desvincula a estrutura de dominação. Uma possível explicação para isso é o tokenismo, fenômeno no qual a inserção da minoria, em grupos onde há a prevalência de grupos dominantes, não possui o mesmo poder, nem a influência do grupo dominante, portanto, não impactando no desempenho (CAMERON, 2001; CAMERON; LALONDE, 2001). Nesse sentido, esforços para o aumento da diversidade e das minorias em posições de conselheiros são necessárias e algumas inclusive em discussão no Brasil, como a questão de cotas para mulheres nos conselhos de empresas estatais e de economia mista.

Nosso estudo contribui para a literatura sobre diversidade dos CAs ao indicar que diversidade, não necessariamente implica em melhor ou pior desempenho, outras características de cunho conjuntural podem ser mais relevantes para indicar desempenho. Esta constatação gera possibilidades de investigações futuras. Do ponto de vista gerencial, este trabalho é relevante para que os gestores compreenderem como as características do CA podem influenciar no desempenho da firma, alertando-os no momento da escolha dos conselheiros.

Reconhecidamente este estudo apresentou limitações. Primeiro a descontinuidade de informações de desempenho de algumas firmas, o que resultou na eliminação destas da 
análise; segundo, o fato de muitas informações contidas no formulário de referência serem auto declaradas e não obrigatórias, limitou a análise de outras características dos conselhos.

Pesquisas futuras podem considerar um recorte longitudinal em que o desempenho possa ser analisado levando em consideração as variáveis macroeconômicas, que podem reconhecidamente influenciar o desempenho das firmas. Além disso, estudos futuros podem utilizar outros indicadores de desempenho para uma análise semelhante. Finalmente, neste estudo, nos concentramos nas firmas brasileiras e, portanto, os achados não podem necessariamente ser generalizados para outros países. Pesquisas futuras podem comparar e contrastar os efeitos da diversidade dos conselhos em diferentes países.

\section{REFERÊNCIAS}

ADAMS, R. B.; FERREIRA, D. Women in the boardroom and their impact on governance and performance. Journal of financial economics, v. 94, n. 2, p. 291-309, 2009. DOI:

https://doi:10.1016/j.jfineco.2008.10.007.

AHMADI, A.; NAKAA, N.; BOURI, A. Chief Executive Officer attributes, board structures, gender diversity and firm performance among French CAC 40 listed firms. Research in International Business and Finance, v. 44, p. 218-226, 2018. DOI:

https://doi.10.1016/j.ribaf.2017.07.083.

AKPAN, E. O.; AMRAN, N. A. Board characteristics and company performance: Evidence from Nigeria. Journal of Finance and Accounting, v. 2, n. 3, p. 81-89, 2014. DOI:

https://doi.10.11648/j.jfa.20140203.17.

ASHBAUGH-SKAIFE, H. A.; COLLINS, D. W.; LAFOND, R. The effects of corporate governance on firms' credit ratings. Available at SSRN 511902, 2004. DOI:

http://doi.org/10.2139/ssrn.511902.

BANGE, M. M.; MAZZEO, M. A. Board composition, board effectiveness, and the observed form of takeover bids. Review of Financial Studies, v. 17, n. 4, p. 1185-1215, 2004. DOI: http://doi.hdl.handle.net/10.1093/rfs/hhh001

BEKANA, D. M.; ABITIE, A. Evaluation of financial performance of banking enterprises: the case of construction and business bank of Ethiopia. Young Economists Journal/Revista Tinerilor Economisti, v. 9, n. 18, p. 82-102, 2012.

BENNOURI, M.; CHTIOUI, T.; NAGATI, H.; NEKHILI, M. Female board directorship and firm performance: What really matters? Journal of Banking \& Finance, 88, 267-291, 2018. DOI: https://doi.10.1016/j.jbankfin.2017.12.010.

BRASIL. Projeto de Lei do Senado n. 112 de 27 de abril de 2010. Diário Oficial da República Federativa do Brasil, Câmara dos Deputados, Brasília, DF, 27 de abril de 2010. Diponível em: https://www25.senado.leg.br/web/atividade/materias/-/materia/96597. Acesso em: 04 dez. 2019.

BRUÈRE, A. J.; MENDES-DA-SILVA, W.; SANTOS, J. F. Aspectos da governança corporativa de empresas listadas na Bovespa: um estudo exploratório sobre a composição e perfil dos 
EDIÇÃO ESPECIAL: COMEMORATIVA AOS 45 ANOS DOS CURSOS DE GRADUAÇÃO EM CIÊNCIAS CONTÁBEIS E ADMINISTRAÇÃO DA UNOCHAPECÓ

conselhos de administração. Revista Base (Administração e Contabilidade) da UNISINOS, v. 4, n. 2, p. 149-159, 2007.

CAMERON, J. E. Social identity, modern sexism, and perceptions of personal and group discrimination by women and men. Sex Roles, v. 45, n. 11-12, p. 743-766, 2001. DOI: https://doi.0360-0025/01/1200-0743/0.

CAMERON, J. E.; LALONDE, R. N. Social identification and gender-related ideology in women and men. British Journal of Social Psychology, v. 40, n. 1, p. 59-77, 2001. DOI: https://doi.10.1348/014466601164696.

CAMPBELL, K.; VERA, A. M. Female board appointments and firm valuation: Short and longterm effects. Journal of Management \& Governance, v. 14, n. 1, p. 37-59, 2010. DOI: https://doi.10.1007/s10997-009-9092-y.

CATALYST. The bottom line: Connecting corporate performance and gender diversity. Catalyst, 2004. Disponível em: https://www.catalyst.org/research/the-bottom-line-connectingcorporate-performance-and-gender-diversity/. Acesso em: 04 dez. 2019.

CATALYST. Australia, Canada, Israel, South Africa, \& United States. Calalyst Quick Takes, 2011. Disponível em: https://www.catalyst.org/publication/239/australia-canada-israel-southafrica-united-states. Acesso em: 04 dez. 2019.

CATALYST. Woman on Corporate Boards Globally. Catalyst Quick Takes, p. 2-15, 2018. Disponível em: https://www.catalyst.org/research/women-on-corporate-boards/. Acesso em: 04 dez. 2019.

CHAU, G.; GRAY, S. J. Family ownership, board independence and voluntary disclosure: Evidence from Hong Kong. Journal of International Accounting, Auditing and Taxation, v. 19, n. 2, p. 93-109, 2010. DOI: https://doi.10.1016/j.intaccaudtax.2010.07.002.

CHEN, J. C.; ROBERTS, R. W. Toward a more coherent understanding of the organizationsociety relationship: A theoretical consideration for social and environmental accounting research. Journal of business ethics, v. 97, n. 4, p. 651-665, 2010. DOI: https://doi.10.1007/s10551-010-0531-0.

DALTON, D. R.; DAILY, C. M.; ELLSTRAND, A. E; JOHNSON, J. L. Meta analytic reviews of board composition, leadership structure, and financial performance. Strategic management journal, v. 19, n. 3, p. 269-290, 1998. DOI: https://doi.org/10.1002/(SICI)1097-0266(199803)19:3<269::AID-SMJ950>3.0.CO;2-K

DARMADI, S. Board diversity and firm performance: the Indonesian evidence. Corporate ownership and control Journal, v. 8, 2011. DOI: https://doi.mpra.ub.unimuenchen.de/38721/.

DEGENHART, L.; VOGT, M.; KAVESKI, I. D. S.; FANK, O. L.; SCARPIN, J. E. Análise dos fatores determinantes do desempenho das empresas familiares brasileiras de capital aberto listadas 
EDIÇÃO ESPECIAL: COMEMORATIVA AOS 45 ANOS DOS CURSOS DE GRADUAÇÃO EM CIÊNCIAS CONTÁBEIS E ADMINISTRAÇÃO DA UNOCHAPECÓ

na BM\&F Bovespa do setor de consumo cíclico. Contexto - Revista do Programa de PósGraduação em Controladoria e Contabilidade da UFRGS, v. 16, n. 33, p. 74-89, 2016.

DIMAGGIO, P. J.; POWELL, W. W. The iron cage revisited: Institutional isomorphism and collective rationality in organizational fields. American sociological review, p. 147-160, 1983. DOI: https://doi.10.2307/2095101.

DUCHIN, R.; MATSUSAKA, J. G.; OZBAS, O. When are outside directors effective? Journal of financial economics, v. 96, n. 2, p. 195-214, 2010. DOI:

https://doi.org/10.1016/j.jfineco.2009.12.004.

ERHARDT, N. L.; WERBEL, J. D.; SHRADER, C. B. Board of director diversity and firm financial performance. Corporate governance: An international review, v. 11, n. 2, p. 102-111, 2003. DOI: https://doi.org/10.1111/1467-8683.00011.

ERTIMUR, Y.; FERRI, F.; STUBBEN, S. R. Board of directors' responsiveness to shareholders: Evidence from shareholder proposals. Journal of Corporate Finance, v. 16, n. 1, p. 53-72, 2010. DOI: https://doi.org/10.1016/j.jcorpfin.2009.07.005.

FAMA, E. F.; JENSEN, M. C. Separation of ownership and control. The journal of law and Economics, v. 26, n. 2, p. 301-325, 1983. DOI:

http://links.jstor.org/sici?sici=0022-

186\%28198306\%2926\%3A2\%3C301\%3ASOOAC\%3E2.0.CO\%3B2-A.

GAUR, S. S.; BATHULA, H.; SINGH, D. Ownership concentration, board characteristics and firm performance: a contingency framework. Management Decision, v. 53, n. 5, p. 911-931, 2015. DOI: https://doi.10.22495/cocv15i1c2p4.

GOES, T. H. M.; MARTINS, H. H.; MACHADO FILHO, C. A. P. Desempenho financeiro de empresas com características familiares: análise de empresas brasileiras listadas na BM\&F. REGE - Revista de Gestão, v. 24, n. 3, p. 197-209, 2017. DOI:

http://dx.doi.org/10.1016/j.rege.2016.06.011.

GUEST, P. M. The determinants of board size and composition: Evidence from the UK. Journal of Corporate Finance, v. 14, n. 1, p. 51-72, 2008. DOI:

https://doi.org/10.1016/j.jcorpfin.2008.01.002.

HAKIMI, A.; RACHDI, H.; MOKNI, R. B. S.; HSSINI, H. Do board characteristics affect bank performance? Evidence from the Bahrain Islamic banks. Journal of Islamic Accounting and Business Research, v. 9, n. 2, p. 251-272, 2018. DOI: https://doi.org/10.1108/JIABR-06-20150029.

HAIR, J. F.; BLACK, W. C.; BABIN, B. J.; ANDERSON, R. E.; TATHAM, R.L. Análise multivariada de dados. Bookman Editora, 2009.

INSTITUTO BRASILEIRO DE GEOGRAFIA E ESTATÍSTICA (IBGE). Estatística de gênero: indicadores sociais das mulheres no Brasil. Estudos e Pesquisas - Informação Demográfica e Socieconômica, v. 38, p. 1-12, 2018. Disponível em: 
EDIÇÃO ESPECIAL: COMEMORATIVA AOS 45 ANOS DOS CURSOS DE GRADUAÇÃO EM CIÊNCIAS CONTÁBEIS E ADMINISTRAÇÃO DA UNOCHAPECÓ

https://biblioteca.ibge.gov.br/visualizacao/livros/liv101551_informativo.pdf. Acesso em: 04 dez. 2019.

INSTITUTO BRASILEIRO DE GOVERNANÇA CORPORATIVA (IBGC). Mulheres no Conselho de Administração, 2009, São Paulo, 2009. Disponível em:

https://conhecimento.ibgc.org.br/Paginas/Publicacao.aspx?Publd=23494. Acessos em: 04 dez. 2019.

INSTITUTO BRASILEIRO DE GOVERNANÇA CORPORATIVA (IBGC). Relatório de Mulheres na Administração das Empresas Brasileiras Listadas - 2010 e 2011, São Paulo, 2011. Disponível em: https://conhecimento.ibgc.org.br/Paginas/Publicacao.aspx?Publd=23495. Acesso em: 04 dez. 2019.

INSTITUTO BRASILEIRO DE GOVERNANÇA CORPORATIVA (IBGC). Perfil dos conselheiros de administração. São Paulo, SP, 2016. Disponível em:

https://conhecimento.ibgc.org.br/Paginas/Publicacao.aspx?Publd=23491. Acesso em: 04 dez. 2019.

JACKLING, B.; JOHL, S. Board structure and firm performance: Evidence from India's top companies. Corporate Governance: An International Review, v. 17, n. 4, p. 492-509, 2009. DOI: https://doi:10.1111/j.1467-8683.2009.00760.x.

JEDI, F. F.; NAYAN, S. An empirical evidence on the effect of women board representation on firm performance of companies listed in Iraq Stock Exchange. Business and Economic Horizons (BEH), v. 14, n. 1232-2019-742, p. 117-131, 2018. DOI:

https://doi:10.15208/beh.2018.10.

JENSEN, M. C. The modern industrial revolution, exit, and the failure of internal control systems. The Journal of Finance, v. 48, n. 3, p. 831-880, 1993. DOI:

https://doi:10.2307/2329018.

JOECKS, J.; PULL, K.; VETTER, K.. Gender diversity in the boardroom and firm performance: What exactly constitutes a "critical mass?". Journal of business ethics, v. 118, n. 1, p. 61-72, 2013. DOI: https://doi:10.1007/s10551-012-1553-6.

KHOSA, A. Independent directors and firm value of group-affiliated firms. International Journal of Accounting \& Information Management, v. 25, n. 2, p. 217-236, 2017. DOI: https://doi:10.1108/IJAIM-08-2016-0076.

KIEL, G. C.; NICHOLSON, G. J. Board composition and corporate performance: How the Australian experience informs contrasting theories of corporate governance. Corporate Governance: An International Review, v. 11, n. 3, p. 189-205, 2003. DOI: https://doi:10.1111/1467-8683.00318.

KONRAD, A. M.; KRAMER, V.; ERKUT, S. The impact of three or more women on corporate boards. Organizational dynamics, v. 37, n. 2, p. 145-164, 2008. DOI: https:

doi:10.1016/j.orgdyn.2008.02.005. 
EDIÇÃO ESPECIAL: COMEMORATIVA AOS 45 ANOS DOS CURSOS DE GRADUAÇÃO EM CIÊNCIAS CONTÁBEIS E ADMINISTRAÇÃO DA UNOCHAPECÓ

LAZZARETTI, K. A participação feminina nos conselhos de administração das empresas brasileiras: uma análise das características de formação e experiência profissional à luz da teoria do capital humano. 2012. Dissertação (Mestrado em Administração) - Universidade do Vale do Itajaí, Biguaçú, 2012.

LAZZARETTI, K. GODOI, C. K.; CAMILO, S. P. O.; MARCON, R. Gender diversity in the boards of directors of Brazilian businesses. Gender in Management: An International Journal, v. 28, n. 2, p. 94-110, 2013. DOI: https://doi:10.1108/17542411311303239.

LEE, L. E.; MARSHAL, R.; RALLIS, D.; MOSCARDI, M. Women on boards: Global trends in gender diversity on corporate boards. MSCI. Nov, 2015. Disponível em: <https://www.msci.com/documents/10199/04b6f646-d638-4878-9c61-4eb91748a82b>. Acesso em: 03 mai. 2018.

LINCK, J. S.; NETTER, J. M.; YANG, T. The determinants of board structure. Journal of financial economics, v. 87, n. 2, p. 308-328, 2008. DOI: https://doi:10.1016/j.jfineco.2007.03.004.

MARTINS, O. S.; MAZER, L. P.; LUSTOSA, P. R. B.; PAULO, E. Características e competências dos conselhos de administração de bancos brasileiros e suas relações com seus desempenhos financeiros (Features and powers of boards of directors of brazilian banks and their relationship with its financial performance). Revista Universo Contábil, v. 8, n. 3, p. 4061, 2012. DOI: https:doi:10.4270/ruc.2012321.

MAHADEO, J. D.; SOOBAROYEN, T.; HANUMAN, V. O. Board composition and financial performance: Uncovering the effects of diversity in an emerging economy. Journal of business ethics, v. 105, n. 3, p. 375-388, 2012. DOI: https://doi:10.1007/s 1055 1-01 1-0973z.

MERCHANT, K. A. Measuring general managers' performances: Market, accounting and combination-of-measures systems. Accounting, Auditing \& Accountability Journal, v. 19, n. 6, p. 893-917, 2006. DOI: https://doi.org/10.1108/09513570610709917.

MEYER, J. W.; ROWAN, B.. Institutionalized organizations: Formal structure as myth and ceremony. American Journal of Sociology, v. 83, n. 2, p. 340-363, 1977. DOI: https://doi:10.1086/226550.

PATHAN, S.; FAFF, R.. Does board structure in banks really affect their performance?. Journal of Banking \& Finance, v. 37, n. 5, p. 1573-1589, 2013. DOI: https://doi:10.1016/j.jbankfin.2012.12.016.

PEARCE, J. A.; ZAHRA, S. A. Board composition from a strategic contingency perspective. Journal of management studies, v. 29, n. 4, p. 411-438, 1992. DOI: https://doi:10.1111/j.1467-6486.1992.tb00672.x. 
EDIÇÃO ESPECIAL: COMEMORATIVA AOS 45 ANOS DOS CURSOS DE GRADUAÇÃO EM CIÊNCIAS CONTÁBEIS E ADMINISTRAÇÃO DA UNOCHAPECÓ

PFEFFER, J.; SALANCIK, G. R. The External Control of Organizations: A Resource Dependence Perspective, Harper \& Row, New York, 1978.

REDDY, K.; LOCKE, S.; SCRIMGEOUR, F.; GUNASEKARAGE, A. Corporate governance practices of small cap companies and their financial performance: An empirical study in Nova Zelândia. International Journal of Business Governance and Ethics, v. 4, n. 1, p. 51-78, 2008. DOI: https://doi.org/10.1504/IJBGE.2008.017891.

SILVA JÚNIOR, C. P.; MARTINS, O. S. Mulheres no conselho afetam o desempenho financeiro? uma análise da representação feminina nas empresas listadas na BM\&FBOVESPA. Sociedade, Contabilidade e Gestão, v. 12, n. 1, 2017. DOI: https://doi.org/10.21446/scg_ufrj.v12i1.13398.

SIMPSON, W. G.; CARTER, D.; D'SOUZA, F. P. What do we know about women on boards? Journal of Applied Finance (Formerly Financial Practice and Education), v. 20, n. 2, p. 27-39, 2010. Disponível em:

https://papers.ssrn.com/sol3/papers.cfm?abstract_id=2693058. Acesso em: 04 dez. 2019.

SINGH, D. A.; GAUR, A. S. Business group affiliation, firm governance, and firm performance: Evidence from China and India. Corporate Governance: An International Review, v. 17, n. 4, p. 411-425, 2009. DOI: https: doi:10.1111/j.1467-8683.2009.00750.x.

TRES, G.; SERRA, F.; FERREIRA, M. P. O tempo de mandato do CEO e o desempenho das empresas: Um estudo comparativo de empresas familiares e não-familiares brasileiras. Revista Gestão \& Tecnologia, v. 14, n. 3, p. 5-31, 2014.

UJUNWA, A. Board characteristics and the financial performance of Nigerian quoted firms. Corporate Governance: The international journal of business in society, v. 12, n. 5, p. 656-674, 2012. DOI: https://doi:10.1108/14720701211275587.

VAN DEN BERGHE, L. A. A; LEVRAU, A. Evaluating boards of directors: what constitutes a good corporate board? Corporate Governance: an international review, v. 12, n. 4, p. 461478, 2004. DOI: https://doi.org/10.1111/j.1467-8683.2004.00387.x.

WAHBA, $\mathrm{H}$. The joint effect of board characteristics on financial performance: Empirical evidence from Egypt. Review of Accounting and Finance, v. 14, n. 1, p. 20-40, 2015. DOI: https://doi:10.1108/raf-03-2013-0029.

WEISBACH, M. S. Outside directors and CEO turnover. Journal of financial Economics, v. 20, p. 431-460, 1988. DOI: https://doi.org/10.1016/0304-405X(88)90053-0

YERMACK, D. Higher market valuation of companies with a small board of directors. Journal of financial economics, v. 40, n. 2, p. 185-211, 1996. DOI: https://doi:10.1016/0304405x(95)00844-5. 